\title{
Atopic dermatitis in South African children: Experience from a tertiary-care centre
}

\author{
o S Katibi, ${ }^{1}$ MBBS, FMCPaed (Nig), MMedSci Derm (SA); N C Dlova, ${ }^{2}$ MB ChB, FCDerm (SA), PhD; \\ A V Chateau, ${ }^{2}$ BSc, MB ChB, DCH, FCDerm (SA), MMedSci Derm (SA); A Mosam, ${ }^{2}$ MB ChB, FCDerm, MMed, PhD (SA) \\ ${ }^{1}$ Department of Paediatrics and Child Health, Faculty of Clinical Sciences, University of Ilorin, Nigeria \\ ${ }^{2}$ Department of Dermatology, Nelson R Mandela School of Medicine, University of KwaZulu-Natal, Durban, South Africa
}

Corresponding author: OS Katibi (oskatibi@gmail.com; katibi.os@unilorin.edu.ng)

\begin{abstract}
Background. Atopic dermatitis (AD) is a chronic, pruritic condition with significant morbidity and some variation in clinical characteristics across different populations.

Objectives. To describe the characteristics and management of $\mathrm{AD}$ in a paediatric cohort.

Methods. A cross-sectional, descriptive study of paediatric AD patients attending the skin clinic at the King Edward VIII Hospital in Durban, South Africa, is described.

Results. The observed prevalence of $\mathrm{AD}$ was $60.1 \%$. A preponderance of male patients (55.6\%) was seen across the cohort. An onset of $\mathrm{AD}$ by 1 year of age was reported in more than half (53.5\%) of the patients. Slightly more than a third of the patients presented with severe disease, as determined by affected body surface area $(\geq 50 \%)$. The head, neck and limbs were the most involved areas. Eczema herpeticum was the most common co-existing dermatosis $(2.4 \%)$. Bleach baths (2\%), wet wrap therapy (3.6\%) and systemic immunosuppressants $(4.8 \%)$ were used in the management of recalcitrant cases.

Conclusion. The observed prevalence of $\mathrm{AD}$ is one of the highest documented among paediatric skin diseases in hospital-based studies in Africa. Predominance in children of preschool age, onset of disease by the age of 5 years, co-existing eczema herpeticum and recalcitrant disease requiring systemic immunosuppressants are consistent with trends in patients from European or Asian descent, while the level of severe disease and head and neck involvement are more consistent with reports from Africa. Further studies in a local context are needed to better understand the disease and its presentation in different populations.
\end{abstract}

S Afr J Child Health 2020;14(4):208-211. https://doi.org/10.7196/SAJCH.2020.v14i4.1718

Atopic dermatitis $(\mathrm{AD})$ is a chronic, relapsing pruritic condition that is associated with significant morbidity and affects a patient's quality of life. It is a disorder that manifests in many ways, ranging from mild disease amenable to treatment with skin emollients to severe disease that requires treatment with systemic immunosuppressants. The aetiology of $\mathrm{AD}$ is characterised by a complex interplay between skin barrier defects, genetic abnormalities, immunologic defects and microbial agents. ${ }^{[1]} \mathrm{AD}$ has been associated with poor sleep, attention deficit hyperactivity disorder and poor school performance, thus revealing the considerable burden of $\mathrm{AD}$ on patients, their families and the society. ${ }^{[2]}$

The prevalence of $\mathrm{AD}$ appears to be increasing worldwide, particularly in developing countries. ${ }^{[3]}$ Although population-based studies are better for studying epidemiological trends, hospitalbased studies are not irrelevant as they can give insight into the burden of diseases, particularly in the healthcare system. Many studies regarding the clinical features and demography of $\mathrm{AD}$ have been described from people of European descent and these features are extrapolated and used globally. For example, flexural dermatitis, which is the typical description of $\mathrm{AD}$, is more likely to manifest in the European American skin than the African American skin, with extensor lesions more commonly seen in the latter group. ${ }^{[4]}$ Similarly, a report by Nnoruka ${ }^{[5]}$ showed a higher prevalence of extensor lesions (70.3\%) in Nigerian patients with $\mathrm{AD}$, particularly among younger patients. Given the variation of features in different populations, it is important to describe $\mathrm{AD}$ in African skin.
Diagnosis of AD is usually clinical, with investigations used to rule out other diagnoses, monitor treatment or confirm the presence of superimposed infections. Management modalities of AD include the use of moisturisers, topical and oral steroids, topical calcineurin inhibitors, phototherapy and systemic immunosuppressants. The mode of management is determined by the severity of the disease, affordability of certain drugs and clinicians' mode of practice. For example, steroid phobia has been described in some parts of the world, but is less common in other regions. ${ }^{[6]}$ With possible differences in the characteristics and management modalities in different populations, the study reported here focuses on $\mathrm{AD}$ in children predominantly of African descent.

\section{Methods}

This was a cross-sectional, descriptive study of children 18 years or younger attending the skin clinic of the King Edward VIII Hospital (a tertiary hospital) in Durban, South Africa (SA), between May and August 2014.

Patients were examined and diagnosed according to the UK Working Party criteria for $\mathrm{AD} \cdot{ }^{[7]}$ Information pertaining to age, gender, age of onset and duration of disease, affected areas, extent of disease, co-existing dermatoses and treatment was obtained. The extent of disease was based on body surface area (BSA) and was recorded as mild ( $<20 \% \mathrm{BSA})$, moderate $(20-49 \% \mathrm{BSA})$ or severe $(\geq 50 \%$ BSA). Data were analysed using SPSS version 20 (IBM Corp., USA). Ethical approval for the study was granted by the University of KwaZulu-Natal (ref. no. BE026/14). 


\section{Results}

Of the 419 children presenting to the clinic with various skin disorders, 252 (60.1\%) were confirmed to have AD based on the mentioned UK criteria. The male-to-female ratio was 1.3:1. Table 1 shows the demographic profile of the study population. Children of preschool age accounted for almost half of the population and a female preponderance was seen in the age group $\geq 6$ years (Table 2). Onset of disease by 1 year of age was reported in more than half (53.5\%) of the children.

The extent of disease was mild in $29.7 \%$ of cases, moderate in $33.8 \%$ and severe in $36.5 \%$. Severe disease was commonly seen in infants, as $52 \%$ had BSA involvement of $\geq 50 \%$. Children of preschool age (1 - 5 years) had the lowest representation of severe AD based on BSA involvement (Fig. 1). Regions of the body affected were the head and neck (80.8\%), limbs (77.9\%) and trunk (51.7\%), and all three these body regions were affected in $40.5 \%$ of the patients. Hand and feet involvement was more commonly seen in children of schoolgoing age and adolescents (14\%) than in children of preschool age $(3.1 \%)$ or infants $(4.0 \%)$.

Itching was the major symptom seen in most patients. Three patients presented with fever and six had significant lymphadenopathy. Table 3 shows the co-existing skin conditions among the cohort and the associated frequencies. Eczema herpeticum was the most common co-existing dermatosis $(n=6,2.4 \%)$. Six patients $(2.4 \%)$ were HIV positive, of whom two presented with co-existing disorders (genital warts and tinea cruris, respectively).

All the patients were treated with emollients and topical steroids. Bleach baths and wet wrap therapy were adjuncts to treatment in $2 \%$ and $3.6 \%$ of cases, respectively. Systemic immunosuppressant treatment was used in 12 patients (4.8\%), of whom 10 were older than 6 years while the remaining two were of preschool age. Azathioprine was used in 10 cases, whereas two patients were treated with cyclosporine. No patient was placed on oral corticosteroids and none were hospitalised.

Table 1. Demographic profile of study population $(N=252)$

\begin{tabular}{ll}
\hline Characteristics & $n(\%)$ \\
\hline Sex & $140(55.6)$ \\
Male & $112(44.4)$ \\
Female & \\
Race & $219(86.9)$ \\
$\quad$ Black African & $29(11.5)$ \\
Indian & $4(1.6)$ \\
Mixed race & \\
Age (months), mean (SD) & 2 weeks - 17 years \\
Range & $47.3(38.7)$ \\
Mean (all) & $54.7(43.5)$ \\
Mean (female) & $41.4(33.3)$ \\
Mean (male) & $23.83(27.06)$ \\
Duration of AD (months), mean (SD) & \\
SD = standard deviation; AD = atopic dermatitis. &
\end{tabular}

Table 2. Age and sex distribution of the study population

\begin{tabular}{llll}
\hline & $\begin{array}{l}\text { Total } \\
(N=252), \\
\text { Age group }\end{array}$ & $\begin{array}{l}\text { Male, } \\
\boldsymbol{n}(\%)\end{array}$ & $\begin{array}{l}\text { Female, } \\
\boldsymbol{n}(\%)\end{array}$ \\
\hline Infants: 0 - 11 months & $41(16.3)$ & $25(61.0)$ & $16(39.0)$ \\
Preschool: 1 - 5 years & $125(49.6)$ & $77(61.6)$ & $48(38.4)$ \\
School age and adolescents: & $86(34.1)$ & $38(44.2)$ & $48(55.8)$ \\
6 - 17 years & & &
\end{tabular}

\section{Discussion}

Although community studies are more representative of the general population, the high prevalence of dermatoses (60.1\%) seen among a paediatric cohort in this hospital-based study suggests that $\mathrm{AD}$ is of considerable magnitude in this population. The observed prevalence is comparable to figures among paediatric patients in a dermatology clinic in Cape Town, SA (59.5\%), as reported by Kakande et al. ${ }^{[8]}$ However, lower prevalences of paediatric skin disorders were found in hospital-based studies in other parts of Africa. For example, Ayanlowo et al. ${ }^{[9]}$ recorded a prevalence of $15.1 \%$ in a densely populated urban region in Lagos, Nigeria, and Kiprono et al. ${ }^{[10]}$ reported a prevalence of $31.5 \%$ from a study in Tanzania. A higher prevalence of paediatric skin disorders in SA has been attributed to higher income levels and increased urbanisation. ${ }^{[11]}$

Among the group as a whole, a slight predominance was seen among male patients, although the prevalence was higher among female patients in the age group $>5$ years. Similar observations have been reported by Verboom et al. ${ }^{[12]}$ and also from a study in Singapore, in which a female predominance was noted in

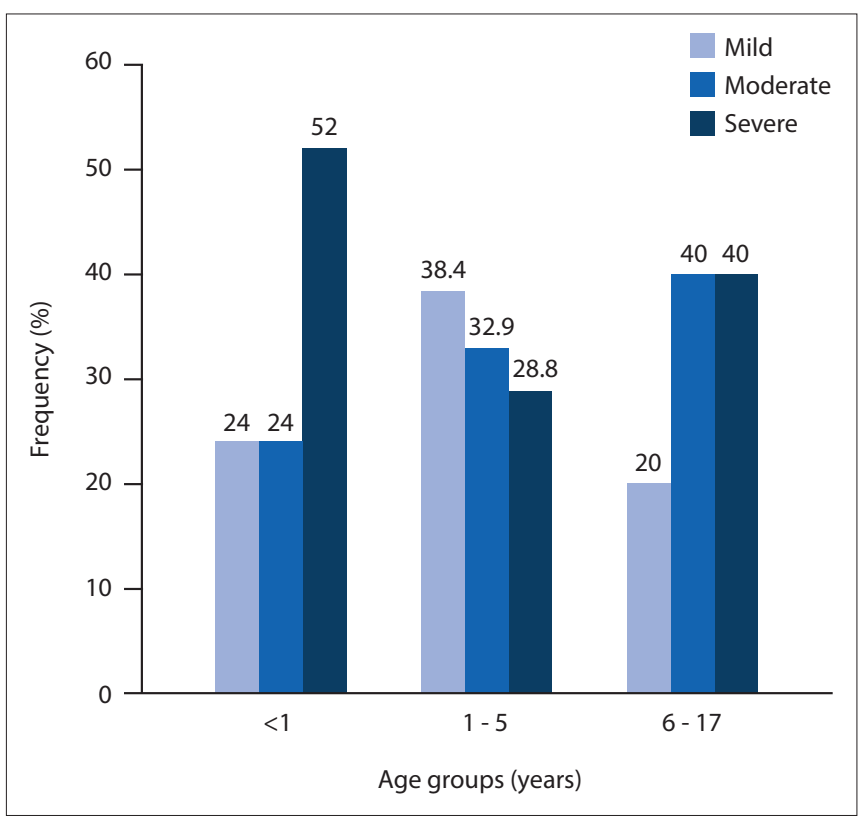

Fig. 1. Extent of disease per age group.

Table 3. Co-existing dermatoses seen in study population $(N=252)$

\begin{tabular}{lc}
\hline Condition & $n(\%)$ \\
\hline Infectious dermatoses & \\
Impetigo & $1(0.4)$ \\
Furunculosis & $1(0.4)$ \\
Tinea corporis & $1(0.4)$ \\
Tinea cruris & $1(0.4)$ \\
Oral candidiasis & $1(0.4)$ \\
Nappy candidiasis & $1(0.4)$ \\
Varicella zoster & $1(0.4)$ \\
Viral warts & $3(1.2)$ \\
Eczema herpeticum & $6(2.4)$ \\
Non-infectious dermatoses & \\
Papular urticaria & $2(0.8)$ \\
Pityriasis alba & $1(0.4)$ \\
Vitiligo & $2(0.8)$
\end{tabular}


16-year-olds compared with a male predominance among 7- and 12-year-old children. ${ }^{[13]}$ Nnoruka $^{[5]}$ reported a female predominance in a Nigerian study that included adults.

Almost half of the patients were between 1 and 5 years old, similar to observations by Horii et al. ${ }^{[14]}$ in a US study and also supported by the findings of Kakande et al.$^{[8]}$ Although AD often develops before 1 year of age, as also seen in this cohort, a delay in diagnosis or parental hesitation to seek treatment during infancy may explain why children of preschool age present to the physician more often than younger children. ${ }^{[14]}$ The large proportion (53.5\%) of children with an onset of $\mathrm{AD}$ by 1 year of age is in keeping with reports of a longitudinal follow-up study by Perkin et al., ${ }^{[15]}$ who found that $33 \%$ of the children followed up had an onset of $\mathrm{AD}$ before 18 months of age, while a later onset was reported in $18 \%$ of cases.

Disease severity varies across populations, as do methods of assessment in different studies. With reference to BSA, which was used to assess the extent of disease in the current study, approximately two-thirds of patients presented with mild or moderate disease. This is less than what has been reported among school-aged children in Singapore, of whom $90 \%$ presented with mild or moderate disease $(\mathrm{BSA}<50 \%){ }^{[13]}$ Nnoruka $^{[5]}$ reported severe $\mathrm{AD}(\mathrm{BSA}>50 \%)$ in $32 \%$ of the patients in that study, similar to our finding of $36.5 \%$. Apart from genetic differences across races and environmental effects in modulating the extent of the disease, patients seeking healthcare in a tertiary centre in this study could account for the larger proportion of cases in which severe extensive disease was seen.

In a study from India, more than $70 \%$ of both the infantile and the childhood AD groups had facial involvement. ${ }^{[16]}$ A similarly large proportion (86\%) of facial dermatitis was reported in a study from China. ${ }^{[17]}$ Involvement of the head and neck (including the face, neck or scalp) was seen in about $80 \%$ of children in our study, in keeping with clinical features of African $\mathrm{AD}$ reported in a recent meta-analysis. ${ }^{[18]}$ It is not unexpected to have found the limbs as a more common site of involvement than the trunk, as flexural and extensor areas of the limbs are known predilection areas for AD. Dermatitis of the hands and feet was more common among the school-aged children and adolescents than in the other age groups, similar to findings from a study in Enugu, Nigeria. ${ }^{[5]}$ Lichenification and post-inflammatory hyperpigmentation were seen in many patients in the current study and have been reported as fairly common in children of African descent, probably owing to frequent rubbing and itching. ${ }^{[4]}$ These features may also be more noticeable on dark skin.

Infectious dermatoses were the most common co-existing disorders, with eczema herpeticum the most prevalent (2.4\%). Eczema herpeticum, a well-known complication of $\mathrm{AD}$ caused by Herpes simplex, occurs because of a complex interplay between host and environmental factors and affects $\sim 3 \%$ of AD patients. ${ }^{[19]} \mathrm{It}$ contributed to a prevalence of $4.0 \%$ of viral disorders in this study (others were viral warts and Varicella zoster infections). Nnoruka ${ }^{[5]}$ reported a prevalence of $2.9 \%$ of viral infections (eczema herpeticum and molluscum contagiosum). Lesions caused by eczema herpeticum are usually found on the limbs and face and progress from vesicular to well-defined excoriated papules or erosions.

All patients with eczema herpeticum in the current study were given oral acyclovir. A propensity for superimposed infection in $\mathrm{AD}$ has been associated with reduced antimicrobial peptides, imbalance in the microbial flora of the skin and a defective skin barrier. ${ }^{[20]}$ In a study from the US, only $24 \%$ of AD patients younger than 2 years were treated with topical steroids and $22 \%$ were treated with topical calcineurin inhibitors; oral steroid use was found in $6 \%$ of children in this age group. ${ }^{[14]}$ This reported use of topical steroids is considerably lower than that observed in our study and may be linked to fear about the long-term effects of steroid use among non-dermatologist physicians in the US, despite it being a well-recognised first-line therapy. ${ }^{[14]}$ Apart from the higher propensity for side-effects such as skin atrophy and growth failure in children, oral steroids are also known to cause rebound flares and as such were not used for any of the patients. Topical calcineurin inhibitors are not used quite as often in our setting as in developed countries owing to high costs; instead, efforts are made to optimise the use of topical steroids through adjunct modalities such as wet wrap therapy.

Research to find the best treatment for $\mathrm{AD}$ is ongoing. Patients with recalcitrant disease, described as 'difficult-to-treat' $\mathrm{AD}$, can be managed with various modalities. In our cohort, wet wrap therapy was used in $3.6 \%$ of cases and bleach baths in $2 \%$ in addition to first-line anti-inflammatory and emollient therapy. At the Mayo Clinic (USA), wet wrap therapy has been reported to be used as a frequent treatment modality in paediatric dermatology patients admitted for $\mathrm{AD} .^{[21]}$ Bleach baths have been shown to be used effectively as a treatment modality in difficult cases, particularly when superimposed Staphylococcus aureus infection is suspected. Huang et al. ${ }^{[22]}$ found a reduction in eczema-affected area and severity index scores with the use of bleach baths and intranasal mupirocin compared with the response in a placebo group. It was also noticed that areas not submerged in bleach, such as the head and neck, did not improve significantly.

Azathioprine was used as a second-line therapy (4\%) in the current study, and cyclosporine as a third-line therapy $(0.8 \%)$. As many physicians are cautious about the use of azathioprine owing to its myelosuppressive effect, full blood counts were monitored monthly for patients so treated in our study. Murphy and Atherton ${ }^{[23]}$ noticed good response with minimal side-effects in children treated with azathioprine once normal erythrocyte thiopurine methyltransferase levels have been established.

In a study from Egypt, El-Khalawany et al. ${ }^{[24]}$ reported similar efficacy and side-effect profiles for treatment with methotrexate and cyclosporine in low doses in their centre. However, methotrexate was not given to any of the patients in the current study. Although treatment with cyclosporine is known to lead to faster resolution of symptoms than with methotrexate or azathioprine, ${ }^{[25]}$ it was used only as a third-line treatment owing to higher costs compared with that of azathioprine.

\section{Study limitations}

As this was a cross-sectional study, information on the seasonal variability of $\mathrm{AD}$ over time was not available. Findings from this hospital-based study may be biased towards patients needing tertiary-level care for their disease.

\section{Conclusion}

This study reports one of the highest prevalences of childhood AD as a form of paediatric dermatosis in a hospital-based study in Africa. Predominance in children younger than 6 years, onset of disease by 5 years of age in most patients, co-existing infectious dermatoses such as eczema herpeticum and recalcitrant disease requiring systemic immunosuppressants seen in about $5 \%$ of patients are consistent with reports from European and Asian populations. Severe disease (based on BSA) seen in a third of this cohort and the head and neck areas being affected to a greater extent are more 
consistent with reports from Africa. The high prevalence and variation in these findings, when compared with other populations within and outside of Africa, may point to genetic or environmental differences that may warrant further study in an effort to reduce the incidence and improve the management of AD. Further studies are needed both locally and elsewhere in Africa to corroborate the findings from the current study.

\section{Declaration. None.}

Acknowledgements. We acknowledge the assistance of the staff of the Dermatology Clinic at the King Edward VIII Hospital.

Author contributions. OSK was the principal investigator and was involved in protocol development, data collection and analysis, and manuscript development. NCD and AM assisted in conceptualisation and design of the study, and review of the protocol, the data collection process and earlier drafts of the manuscript. AVC contributed to data collection and interpretation and manuscript development. All authors approved the final version.

\section{Funding. None.}

Conflicts of interest. None.

1. Peng W, Novak N. Pathogenesis of atopic dermatitis. Clin Exp Allergy 2015;45(3):566-574. https://doi.org/10.1111/cea.12495

2. Camfferman D, Kennedy JD, Gold M, Martin AJ, Winwood P, Lushington K. Eczema, sleep, and behavior in children. J Clin Sleep Med 2010;6(06):581-588. https://doi.org/10.5664/jcsm.27992

3. Deckers IA, McLean S, Linssen S, Mommers M, Van Schayck CP, Sheikh A. Investigating international time trends in the incidence and prevalence of atopic eczema 1990-2010: A systematic review of epidemiological studies. PLoS One 2012;7(7):e39803. https://doi.org/10.1371/journal.pone.0039803

4. Kaufman BP, Guttman-Yassky E, Alexis AF. Atopic dermatitis in diverse racial and ethnic groups -variations in epidemiology, genetics, clinical presentation and treatment. Exp Dermatol 2018;27(4):340-357. https://doi.org/10.1111/ exd. 13514

5. Nnoruka EN. Current epidemiology of atopic dermatitis in south-eastern Nigeria. Int J Dermatol 2004;43(10):739-744. https://doi.org/10.1111/j.13654632.2004.02360.x

6. El Hachem M, Gesualdo F, Ricci G, et al. Topical corticosteroid phobia in parents of pediatric patients with atopic dermatitis: A multicentre survey. Ital J Pediatr 2017;43(1):22. https://doi.org/10.1186/s13052-017-0330-7

7. Williams HC, Burney PG, Hay RJ, et al. The U.K. Working Party's Diagnostic Criteria for Atopic Dermatitis. I. Derivation of a minimum set of discriminators for atopic dermatitis. Br J Dermatol 1994;131(3):383-396. https://doi. org/10.1111/j.1365-2133.1994.tb08530.x

8. Kakande B, Gumedze F, Hlela C, Khumalo NP. Focus on the top ten diagnoses could reduce pediatric dermatology referrals. Pediatr Dermatol 2016;33(1):99102. https://doi.org/10.1111/pde.12714
9. Ayanlowo O, Puddicombe O, Gold-Olufadi S. Pattern of skin diseases amongst children attending a dermatology clinic in Lagos, Nigeria. Pan Afr Med J 2018;29(1):1-10. https://doi.org/10.11604/pamj.2018.29.162.14503

10. Kiprono SK, Muchunu JW, Masenga JE. Skin diseases in pediatric patients attending a tertiary dermatology hospital in Northern Tanzania: A crosssectional study. BMC Dermatol 2015;15(1):16. https://doi.org/10.1186/s12895015-0035-9

11. Zar HJ, Ehrlich RI, Workman L, Weinberg EG. The changing prevalence of asthma, allergic rhinitis and atopic eczema in African adolescents from 1995 to 2002. Pediatr Allergy Immunol 2007;18(7):560-565. https://doi.org/10.1111/ j.1399-3038.2007.00554.x

12. Verboom P, Hakkaart-Van Roijen L, Sturkenboom M, De Zeeuw R, Menke H, Rutten F. The cost of atopic dermatitis in the Netherlands: An international comparison. Br J Dermatol 2002;147(4):716-724. https://doi.org/10.1046/j.13652133.2002.04964.X

13. Tay Y-K, Kong K-H, Khoo L, Goh C-L, Giam Y-C. The prevalence and descriptive epidemiology of atopic dermatitis in Singapore school children. Br J Dermatol 2002;146(1):101-106. https://doi.org/10.1046/j.1365-2133.2002.04566.x

14. Horii KA, Simon SD, Liu DY, Sharma V. Atopic dermatitis in children in the United States, 1997-2004: Visit trends, patient and provider characteristics, and prescribing patterns. Pediatrics 2007;120(3):e527-e534. https://doi.org/10.1542/ peds.2007-0289

15. Perkin MR, Strachan DP, Williams HC, Kennedy CT, Golding J, ALSPAC Study Team. Natural history of atopic dermatitis and its relationship to serum total immunoglobulin E in a population-based birth cohort study. Pediatr Allergy Immunol 2004;15(3):221-229. https://doi.org/10.1111/j.1399-3038.2004.00160.x

16. Dhar S, Kanwar AJ. Epidemiology and clinical pattern of atopic dermatitis in a North Indian pediatric population. Pediatr Dermatol 1998;15(5):347-351. https://doi.org/10.1111/j.1525-1470.1998.tb01362.x

17. Shi M, Zhang H, Chen X, et al. Clinical features of atopic dermatitis in a hospitalbased setting in China. J Eur Acad Dermatol Venereol 2011;25:1206-1212. https://doi.org/10.1111/j.1468-3083.2010.03953.x

18. Yew YW, Thyssen JP, Silverberg JI. A systematic review and meta-analysis of the regional and age-related differences in atopic dermatitis clinical characteristics. J Am Acad Dermatol 2019;80(2):390-401. https://doi.org/10.1016/j. jaad.2018.09.035

19. Leung DYM. Why is eczema herpeticum unexpectedly rare? Antiviral Res 2013;98(2):153-157. https://doi.org/10.1016/j.antiviral.2013.02.010

20. Sun D, Ong PY. Infectious complications in atopic dermatitis. Immunol Allergy Clin North Am 2017;37(1):75-93. https://doi.org/10.1016/j.iac.2016.08.015

21. Storan ER, McEvoy MT, Wetter DA, et al. Pediatric hospital dermatology: Experience with inpatient and consult services at the Mayo Clinic. Pediatr Dermatol 2013;30(4):433-437. https://doi.org/10.1111/pde.12081

22. Huang JT, Rademaker A, Paller AS. Dilute bleach baths for Staphylococcus aureus colonisation in atopic dermatitis to decrease disease severity. Arch Dermatol 2011;147(2):246-247. https://doi.org/10.1001/archdermatol.2010.434

23. Murphy L-A, Atherton D. A retrospective evaluation of azathioprine in severe childhood atopic eczema, using thiopurine methyltransferase levels to exclude patients at high risk of myelosuppression. Br J Dermatol 2002;147(2):308-315. https://doi.org/10.1046/j.1365-2133.2002.04922.x

24. El-Khalawany MA, Hassan H, Shaaban D, Ghonaim N, Eassa B. Methotrexate versus cyclosporine in the treatment of severe atopic dermatitis in children: A multicenter experience from Egypt. Eur J Pediatr 2013;172(3):351-356. https:// doi.org/10.1007/s00431-012-1893-3

25. Sidbury R, Hanifin J. Systemic therapy of atopic dermatitis. Clin Exp Dermatol 2000;25(7):559-566. https://doi.org/10.1046/j.1365-2230.2000.00697.x

Accepted 23 August 2020. 